\title{
A new semi-selective agar medium for recovery and enumeration of antagonistic yeast, Pichia guilliermondii strain Z1 from orange fruit surface
}

\author{
Rachid Lahlali ${ }^{1,4 \star}$, Younes Hamadi ${ }^{2,3}$, Mohamed El Guilli ${ }^{3}$ and M. Haissam Jijakli ${ }^{1}$ \\ ${ }^{1}$ Plant Pathology Unit, Gembloux Agro-Bio Tech, University of Liege, Passage des Deportes, 2, 5030 gembloux, \\ Belgium. \\ ${ }^{2}$ Universite Ibn Tofail, Faculté des Sciences, Laboratoire de Phytopathologie, BP 133, 14000 Kenitra, Morocco. \\ ${ }^{3}$ INRA-El Menzeh, Laboratoire de Phytopathologie, BP 293, 14000 Kenitra, Morocco. \\ ${ }^{4}$ AAFC-Saskatoon Research Centre, 107 Science Place, Saskatchewan, S7N0X2, Canada.
}

Accepted 17 June, 2013

\begin{abstract}
The aim of this work was to develop a semi-selective medium for recovery and enumeration of Pichia guilliermondii strain $\mathrm{Z1}$, a reliable biocontrol agent against postharvest pathogens of citrus fruit, and to assess the population dynamic of this antagonistic yeast on orange fruit in relation to incubation temperature and time of incubation. PDA is the basal medium used in this study which allows the antagonistic strain Z1 source of carbon and nutrients. Different chemicals (thiophanate-methyl, thiabendazole, thiram and imazalil) and antibiotics (hygromycin, tetracyclin, ampicillin and chloramphenicol) have been tested individually and based on the plating efficiency up to $90 \%, 40$ combinations have been assayed between antibiotics and fungicides. Ten combinations proved to be highly selective against citrus pathogens and laboratory microflora, but only one consisting of tetracyclin $(1 \mathrm{~g} / \mathrm{L})$ and thiabendazole $(60 \mathrm{mg} / \mathrm{L})$ was retained based on plating efficiency up to $99 \%$, total selectivity against laboratory microflora, epiphytic microflora from washed orange fruit and lower cost. The semi-selective medium TET-TBZ-PDA has been used to assess the impact of temperature and incubation time on the survival of strain Z1. It appears that population density was significantly influenced by both factors and the highest population size was recorded at $25^{\circ} \mathrm{C}$ followed by 5 and $35^{\circ} \mathrm{C}$, respectively. This strain required a time of adaptation before entering the exponential growth phase with a maximum growth observed at $25^{\circ} \mathrm{C}$ relative to others. The semi-selective medium TETTBZ-PDA could be an efficient and valuable way to track the population density of this strain on the surface of orange when applied pre-or postharvest. This semi-selective medium may also aid in reaching a population density allowing a better efficiency in relation to environmental conditions.
\end{abstract}

Key words: Antibiotics, biocontrol, citrus, fungicides, quantification, plating efficiency, Pichia guilliermondii strain Z1, semi-selective medium.

\section{INTRODUCTION}

Morocco is one of the largest exporting countries of citrus fruit, after Turkey, South Africa, USA and Spain (Citrus commodity, 2005; Lahlali et al., 2011). Wound pathogens of citrus fruit are responsible for numerous losses worldwide (Zhang et al., 2005). Penicillium sp. and Geotrichum species are the most common and serious 
diseases that affect citrus fruit quality in Mediterranean climates (Lahlali et al., 2004; Palou et al., 2002). In Morocco, the losses have been estimated at over $60 \%$ and are generally controlled by applying chemical fungicides before or after harvesting (Lahlali et al., 2004, 2005; Taqarort et al., 2008). However, the usual appearance of strains resistant to chemicals, the increased concern for the environment and human health, and public demand for minimal residues have contributed to the development of alternative strategies to substitute the use of synthetic chemicals.

Pichia guilliermondii strain $\mathrm{Z1}$, is an effective biocontrol agent against citrus fruit diseases in Morocco (Lahlali et al., 2011). This strain have been isolated from the surface of orange, formulated and then tested against major postharvest citrus diseases such as Penicillium italicum and Penicillium digitatum (El Guilli et al., 2012; Lahlali et al., 2011). The monitoring of antagonistic yeasts has been well developed and consists of two principal methods; specific detection using molecular tools and plating methods (De Clercq et al., 2003). The monitoring of strain $\mathrm{Z} 1$ requires the development of a specific method that is able to quantify the population of the biocontrol agent $(\mathrm{BCA})$ and to distinguish it from the indigenous micro flora. As the protective effect of strain Z1 is closely related to colonization (El Guilli et al., 2012), evaluating the ecological fitness of $P$. guilliermondii strain $\mathrm{Z} 1$ after treatment on citrus is critical for the interpretation and prediction of its biocontrol efficacy in relation to several parameters (methods of BCA application, environmental conditions of storage rooms, integration of BCA to other sanitary measures). Finally, this method will be used to fulfil some eco-toxicological requirements for the registration procedure. In this context, the objective of this work was to develop a semi-selective medium to monitor the population of the biocontrol yeast on citrus fruit where there might be the presence of spoilage fungi as well as epiphytic/spoilage bacteria; this medium need to be formulated with agents to inhibit the growth of the spoilage fungi (antifungal agents) and bacteria (antibiotics) without inhibiting the growth of antagonistic yeast strain Z1. The formulation of such medium using a combination of chemical fungicide and antibiotics and its performance was discussed in the present work. Such study is of great importance and permits correlation of efficiency with population size and determines the concentration of this yeast that should be applied to reach a higher reduction of disease incidence due to wound pathogens.

\section{MATERIALS AND METHODS}

\section{Micro-organisms preparation}

Yeast strain Z1 was isolated from healthy Moroccan Valencia late oranges by the laboratory of Phytopathology of INRA-El Menzeh and identified as $P$. guilliermondii. It was selected for its high and reliable protective activity against $P$. italicum and $P$. digitatum (El
Guilli et al., 2009; Lahlali et al., 2011). Before use, it was subcultured at $25^{\circ} \mathrm{C}$ for three successive generations on Potato dextrose agar (PDA, Merck, Darmstadt, Germany) medium with an interval of $24 \mathrm{~h}$. The final concentration was determined by photo spectrometer following the equation (OD- 0.0958) $/ 0.03=\mathrm{CFU} / \mathrm{mL} \times$ $\left.10^{6}\right)$.

$P$. italicum and $P$. digitatum strains used in this study were originnally isolated from decayed orange fruit from the Gharb region of Morocco (El Guilli et al., 2012). For long term storage, both pathogens were maintained in $25 \%$ glycerol at $-80^{\circ} \mathrm{C}$. Both fungus were recovered from glycerol and grown on potato dextrose agar (PDA) when they were needed for selectivity of semi-selective medium test.

\section{Media preparation and compound screening}

For the development of a semi-selective medium to track the population of $P$. guilliermondii strain Z1, 4 fungicides, thiophanatemethyle (Tpm), thiabendazole (TBZ), thiram (Tm) and imazalil (Im) and 4 antibiotics hygromycin (Hy), tetracyclin (TET), ampicillin (Am) and chloramphenicol (Ch) were evaluated at different concentrations using PDA as the basal medium. The use of PDA medium ensures the requirement of strain Z1 for nutrients and carbon. These antifungal and antibiotic agents were selected based on their availability in our laboratory. Each compound was tested individually before proceeding to testing combinations. The media were separately supplemented with fungicide substances after cooling at $50^{\circ} \mathrm{C}$. The mixture (substance plus fungicide) was then homogenized by manual shaking for $30 \mathrm{~s}$ and then poured onto Petri dishes under aseptic conditions. Similarly, antibiotics were tested individually at five concentrations each. Each antibiotic was incurporated into PDA media after cooling to $50^{\circ} \mathrm{C}$. Media were homogenized by shaking for $30 \mathrm{~s}$. Five concentrations were tested for each PDA-antibiotic or fungicide combination (Tables 1 and 2).

The toxicity of fungicide and antibiotics media was first evaluated by plating $2 \times 10^{2} \mathrm{CFU}$ on each media test as well as on PDA without compound. Concentration $(\mathrm{CFU} / \mathrm{mL})$ of strain $\mathrm{Z1}$ was determined using the Burker cell. Plate dishes were incubated at $25^{\circ} \mathrm{C}$ for 3 to 7 days and then the number of colony forming units (CFU) and plating efficiency was reported. This experiment was repeated twice with four replicates.

\section{Fungicides and antibiotics combination test}

This experiment also tested which fungicide and antibiotic combination was less toxic for strain Z1. After evaluating fungicides and antibiotics individually, different combinations were evaluated between selected concentrations of fungicides and antibiotics (more than 40 combinations) using the same test above and only those given a better plating efficiency were retained for a selectivity test against postharvest pathogens of citrus, air laboratory microflora and epiphytic mircoflora of orange surface. Four Petri dishes were plated for each combination. This experiment was repeated twice.

\section{Toxicity and selectivity of semi-selective media}

Ten microlitres of $P$. italicum or $P$. digitatum suspensions at concentration of $1 \times 10^{5}$ spores $/ \mathrm{mL}$, were placed at the centre of each semi selective medium. Petri plates were then incubated at $25^{\circ} \mathrm{C}$ for 7 days. To evaluate the selectivity of the different chosen media against laboratory air microflora, three Petri dishes per medium, including PDA, were left open for $3 \mathrm{~h}$ on the laboratory bench.

The toxicity and selectivity of selected semi-selective medium was evaluated on strain Z1 yeast cells on the surface of oranges. Twelve 'Valencia-late' fruit were dipped in a water suspension containing antagonistic yeast at $1 \times 10^{7} \mathrm{CFU} / \mathrm{ml}$ for $2 \mathrm{~min} ; 12$ 
Table 1. Plating efficiency (\%) of $P$. guilliermondii on potato dextrose agar (PDA) amended with fungicides.

\begin{tabular}{|c|c|c|c|}
\hline Fungicides (active substances) & Medium code & Compound $^{\text {a }}$ concentration (mg/L) & Plating efficiency $(\%)^{\mathrm{b}}$ \\
\hline \multirow{5}{*}{ Fungaflor (Imazalil) } & $\operatorname{Im} 01$ & $\operatorname{Im}(0.1)$ & $93.07^{\mathrm{a}}$ \\
\hline & $\operatorname{Im} 02$ & $\operatorname{Im}(0.5)$ & $91.05^{\mathrm{a}}$ \\
\hline & $\operatorname{Im} 03$ & $\operatorname{Im}(1)$ & $90.9^{a}$ \\
\hline & Im04 & $\operatorname{Im}(5)$ & $82.5^{\mathrm{ab}}$ \\
\hline & Im05 & $\operatorname{Im}(10)$ & $74.19^{\mathrm{ab}}$ \\
\hline \multirow{5}{*}{ TMTD (Thiram) } & Tm01 & $\operatorname{Tm}(0.1)$ & $90.07^{\mathrm{a}}$ \\
\hline & Tm02 & $\operatorname{Tm}(0.5)$ & $73.97^{b}$ \\
\hline & Tm03 & $\operatorname{Tm}(1)$ & $61.31^{b}$ \\
\hline & Tm04 & $\operatorname{Tm}(5)$ & $19.00^{c}$ \\
\hline & Tm05 & $\operatorname{Tm}(10)$ & $5.79^{c}$ \\
\hline \multirow{5}{*}{ Lirotect (Thiabendazole) } & TBZ01 & TBZ (0.5) & $95.91^{\mathrm{a}}$ \\
\hline & TBZ02 & TBZ (1) & $92.09^{\mathrm{a}}$ \\
\hline & TBZ03 & TBZ (10) & $92.58^{\mathrm{a}}$ \\
\hline & TBZ04 & TBZ (60) & $96.35^{\mathrm{a}}$ \\
\hline & TBZ05 & TBZ (80) & $81.5^{\mathrm{ab}}$ \\
\hline \multirow{5}{*}{ Topsin (Thiophanate-methyl) } & Tpm01 & $\operatorname{Tpm}(0.5)$ & $91.76^{\mathrm{a}}$ \\
\hline & Tpm02 & Tpm (1) & $90.62^{\mathrm{a}}$ \\
\hline & Tpm03 & Tpm (5) & $86.74^{\mathrm{ab}}$ \\
\hline & Tpm04 & Tpm (10) & $81.12^{\mathrm{ab}}$ \\
\hline & Tpm05 & Tpm (80) & $16.54^{\mathrm{c}}$ \\
\hline
\end{tabular}

${ }^{\mathrm{a}}$ Imazalil (Im), TMTD (Tm), thiabendazole (TBZ), thiophanate-methyl (Tpm). ${ }^{b}$ Efficiency $(\%)=[(\mathrm{CFU}$ on the tested medium)/(CFU on PDA medium)] $\times 100$.

orange fruit remained untreated. After drying at ambient temperature for 2 h, 4 fruit from treated and untreated orange were washed separately in KBPT buffer [(KH2PO4 (0.05M), K2HPO4 (0.05M) and $0.05 \%$ of Tween $80, \mathrm{pH} 6.5$ )] for $20 \mathrm{~min}$ on a rotatory shaker (Gerhardt, Germany) at $120 \mathrm{rpm}$ for $20 \mathrm{~min}$. After washing, $5 \mathrm{ml}$ of wash water sample were collected and serially diluted 10 fold. One hundred microlitres from each washing replicate was plated out in triplicate on each semi-selective test medium and PDA. Petri dishes were incubated at $25^{\circ} \mathrm{C}$ for 4 days and white colonies morphologically similar to strain Z1 were counted. This experiment was repeated twice with triplicates. The result was evaluated in two ways: plating efficiency (\%) and by qualitative observations of the natural microorganisms occurring on washing orange surfaces on each semi-selective medium test in comparison with PDA medium.

\section{Impact of temperature on the survival of strain Z1 on orange} fruit

The same procedure was used to evaluate the impact of temperature on strain Z1 survival on orange fruit surface. The recovery of strain Z1 population from orange fruits stored at three temperatures 5,25 and $35^{\circ} \mathrm{C}$ was done by using the semi-selective medium TETTBZ-PDA. The strain Z1 recovery was evaluated daily on treated orange fruit and results were reported as number of colony forming units per $\mathrm{cm}^{2}$ of orange surface using the following equation:

Surface $=1.024 \times$ Weight $+27.66\left(R^{2}=0.96\right)$. This experiment was repeated twice in triplicate.

\section{Statistical analysis}

Plating efficiency (\%) was calculated according to the formula: Efficiency $(\%)=[($ CFU on the tested medium/(CFU on PDA medium $)] \times$ 100 and then transformed to square root before subjected to ANOVA using SAS software (version 9.1, SAS Institute Inc. Gary, $\mathrm{NC}$ ). When the effect was revealed to be significant, an LSD test was used for means separation.

\section{RESULTS}

\section{Fungicide and antibiotic screening}

According to the toxicity test (plating efficiency), concentrations of fungicides and antibiotics showing higher efficiency up to $90 \%$ (Tables 1 and 2) were retained for evaluation in semi-selective medium ingredients. Table 1 shows that the highest non-toxic concentrations for strain Z1 were obtained with thiabendazole $(60 \mathrm{mg} / \mathrm{L})$, imazalil $(1 \mathrm{mg} / \mathrm{L})$, thiophanate-methyl $(1 \mathrm{mg} / \mathrm{L})$ and thiram $(0.1$ $\mathrm{mg} / \mathrm{L})$. However, in the case of antibiotics, tetracyclin (1 $\mathrm{g} / \mathrm{L})$ appears to be non-toxic for Z1 followed respectively by ampicillin $(500 \mathrm{mg} / \mathrm{L})$, chloramphenicol $(10 \mathrm{mg} / \mathrm{L})$ and hygromycin $B(5 \mathrm{mg} / \mathrm{L})$ (Table 2$)$. These different active substances, which have a different mode of action, could be used in combination in order to increase the toxicity 
Table 2. Plating efficiency (\%) of $P$. guilliermondii on PDA amended with antibiotics.

\begin{tabular}{|c|c|c|c|}
\hline Antibiotic & Medium code & Compound $^{\mathrm{a}}$ concentration $(\mathrm{mg} / \mathrm{L})$ & Plating efficiency $(\%)^{\mathrm{b}}$ \\
\hline \multirow{5}{*}{ Hygromycin B } & $\mathrm{Hy} 01$ & Hy (5) & $90.20^{\mathrm{ab}}$ \\
\hline & $\mathrm{Hy} 02$ & Hy (10) & $33.20^{d}$ \\
\hline & $\mathrm{HyO3}$ & Hy (50) & $3.08^{\mathrm{e}}$ \\
\hline & $\mathrm{Hy} 04$ & Hy (100) & $1.23^{\mathrm{e}}$ \\
\hline & Hy05 & Hy (250) & $1.05^{\mathrm{e}}$ \\
\hline \multirow{5}{*}{ Tetracyclin } & Tet01 & Tet $(50)$ & $92.83^{\mathrm{ab}}$ \\
\hline & Tet02 & Tet (100) & $100^{\mathrm{a}}$ \\
\hline & Tet03 & Tet $(250)$ & $100^{\mathrm{a}}$ \\
\hline & Tet04 & Tet $(500)$ & $92.70^{\mathrm{ab}}$ \\
\hline & Tet05 & Tet $(1000)$ & $100^{\mathrm{a}}$ \\
\hline \multirow{5}{*}{ Ampicillin } & Am01 & $\operatorname{Am}(10)$ & $92.18^{\mathrm{ab}}$ \\
\hline & Am02 & Am (50) & $90.20^{a b}$ \\
\hline & $\mathrm{Am03}$ & Am (100) & $95.30^{\mathrm{ab}}$ \\
\hline & Am04 & Am (250) & $82.70^{\mathrm{bc}}$ \\
\hline & Am05 & Am (500) & $99.36^{\mathrm{a}}$ \\
\hline \multirow{5}{*}{ Chloramphenicol } & Ch01 & Ch (5) & $91.03^{\mathrm{ab}}$ \\
\hline & Ch02 & Ch (10) & $90.39^{\mathrm{ab}}$ \\
\hline & Ch03 & Ch (100) & $85.65^{\mathrm{bc}}$ \\
\hline & Ch04 & Ch (250) & $84.28^{\mathrm{bc}}$ \\
\hline & Ch05 & Ch (500) & $75.52^{c}$ \\
\hline
\end{tabular}

${ }^{\mathrm{a}}$ Hygromycin B $(\mathrm{Hy})$, tetracyclin (Tet), ampicillin $(\mathrm{Am})$ and chloramphenicol $(\mathrm{Ch})$. ${ }^{\mathrm{b}}$ Efficiency $(\%)=[(\mathrm{CFU}$ on the tested medium $) /($ CFU on PDA medium $)] \times 100$.

of medium against exogenous micro-organisms without disturbing the strain $\mathrm{Z1}$ viability (viability $\geq 90 \%$ ).

\section{Combinations of fungicides and antibiotics test}

Different combinations (40 in total) were assayed from selected active substances imazalil, thiophanate-methyl, thiram, thiabendazole, hygromycin B, tetracyclin, ampicillin and chloramphenicol (data not shown). The most important among them and which showed a plating efficiency up to $90 \%$ are listed in Table 3 (at least 20 combinations). All combinations were non-toxic for strain Z1, except in the case when hygromycin was added. It has been noticed that a selective medium that contains more non-toxic active ingredients for strain Z1, always gives a large spectrum of action and can prevent the development of all pathogens. However, given the high cost of antibiotics and fungicides, and the need of easier semiselective medium for routine preparation and use, we have attempted to reduce the active ingredients of semiselective medium. Semi-selective medium which permit easier recovery of strain Z1 from orange surface and which are selective for most postharvest citrus diseases and other air laboratory microorganisms was considered. Results from different assays are summarized in Table 3, in which the combination containing tetracyclin $(1 \mathrm{~g} / \mathrm{mL})$ and thiabendazole $(60 \mathrm{mg} / \mathrm{mL})$ have inhibited the growth of fungal pathogens and other micro-organisms. This combination has been chosen as a semi-selective medium for recovery and enumeration of antagonistic strain Z1 among other effective combinations.

\section{Toxicity and selectivity of semi-selective media}

To validate the obtained results, the potential toxicity of the semi-selective media was assessed (Table 3). Among the tested media, TET-TBZ-PDA was chosen. This semiselective medium was first evaluated by directly plating strain Z1 on that medium according to the incubation time. The number of colony forming unit (CFU/dish) were observed on PDA and on semi-selective medium between 3 and 7 days incubation time. Table 4 shows clearly that selected semi-selective medium TET-TBZ-PDA was non-toxic to strain Z1 with similar number of CFU on both media, whatever the incubation time may be. No delayed colony development was observed on TET-TBZ-PDA between 3 and 7 days of incubation at $25^{\circ} \mathrm{C}$.

The selectivity of the medium was also evaluated by comparison of natural occurring microorganisms grown on PDA and TET-TBZ-PDA after plating of KPB buffer washed surfaces of untreated and treated oranges (Figure 1). While the PDA medium was covered with 
Table 3. Plating efficiency (\%) and selectivity of semi-selective media amended with antibiotics and fungicides.

\begin{tabular}{lccccc}
\hline Medium combination & Washing water & Ambient air & $\boldsymbol{P}$. italicum & P. digitatum & Plating efficiency (\%) \\
\hline Tm01+TET05 & - & - & - & - & $97.70^{\mathrm{ab}}$ \\
Im03+TET05 & - & - & - & - & $93.85^{\mathrm{ab}}$ \\
TBZ04+TET05 & - & - & - & - & $100^{\mathrm{a}}$ \\
Tpm01+TET05 & - & + & - & + & $98.08^{\mathrm{a}}$ \\
Tm01+Am05 & - & - & - & - & $98.85^{\mathrm{a}}$ \\
TBZ04+Am05 & - & + & - & - & $99.24^{\mathrm{a}}$ \\
Im03+Am05 & - & + & - & + & $94.24^{\mathrm{ab}}$ \\
Tpm01+Am05 & - & + & + & + & $96.16^{\mathrm{ab}}$ \\
Tm01+Ch02 & - & + & - & - & $96.54^{\mathrm{ab}}$ \\
TBZ04+Ch02 & + & + & - & - & $97.85^{\mathrm{ab}}$ \\
Im01+Ch02 & - & + & - & - & $95^{\mathrm{ab}}$ \\
Tpm01+Ch02 & + & + & + & + & $99.62^{\mathrm{a}}$ \\
TBZ04+Tm01+Am05 & - & - & - & - & $91.93^{\mathrm{ab}}$ \\
TBZ04+Tm01+TET05 & - & - & - & - & $99.24^{\mathrm{a}}$ \\
Im01+Tm01+Am05 & - & - & - & - & $92.7^{\mathrm{ab}}$ \\
Im01+Tm01+TET05 & - & - & - & - & $98.08^{\mathrm{a}}$ \\
Im01+Tm01+Ch02 & - & - & - & - & $95.77^{\mathrm{ab}}$ \\
Tm01+Am05+TET05 & - & - & - & - & $98.85^{\mathrm{a}}$ \\
Tm01+Am05+Ch02 & - & + & - & - & $91.54^{\mathrm{ab}}$ \\
Tm01+Am05+Ch02 & - & - & - & - & $96.93^{\mathrm{ab}}$ \\
\hline
\end{tabular}

Tetracyclin (TET), ampicillin (Am) and chloramphenicol (Ch), imazalil (Im), TMTD (Tm), thiabendazole (TBZ), thiophanatemethyl (Tpm), (-) absence and (+) presence of contaminants. ${ }^{b}$ Efficiency $(\%)=[($ CFU on the tested medium) $/($ CFU on PDA medium) $] \times 100$.

Table 4. Analysis of toxicity effects of the semi-selective medium TET-TBZ-PDA on the growth of $P$. guilliermondii strain Z1 (CFU/dish) as compared to the PDA medium.

\begin{tabular}{lcccc}
\hline \multirow{2}{*}{ Media } & \multicolumn{4}{c}{ Incubation time (days) } \\
\cline { 2 - 5 } & $\mathbf{3}$ & $\mathbf{4}$ & $\mathbf{5}$ & $\mathbf{7}$ \\
\hline PDA & $255 \pm 20$ & $257 \pm 18$ & $257 \pm 18$ & $257 \pm 18$ \\
TET-TBZ-PDA & $241 \pm 8$ & $263 \pm 6$ & $264 \pm 5$ & $264 \pm 5$ \\
\hline
\end{tabular}

yeast, mycelial fungi and bacteria, the TET-TBZ-PDA medium only showed the development of white colonies morphologically similar to strain Z1.

\section{Impact of temperature on the survival of strain Z1 on orange fruit}

The impact of temperature on the survival of strain $\mathrm{Z1}$ has been assessed on orange fruit surface using the developed semi-selective medium TET-TBZ-PDA at three incubation temperatures: 5,25 and $35^{\circ} \mathrm{C}$. Statistical analysis showed a significant effect of time and incubation temperature of the survival of strain Z1 (data not shown). Figure 2 shows that, during the first $3 \mathrm{~h}$, the strain $\mathrm{Z1}$ cell numbers were constant $\left(1 \times 10^{4} \mathrm{CFU} / \mathrm{cm}^{2}\right)$ and were shown to be unaffected by the incubation time and then increased exponentially to reach the plateau phase of $261.5 \%$ of viability after $24 \mathrm{~h}$ of incubation time. Cell numbers were approximately three times the initial concentration. The higher strain Z1 density was obtained with incubation temperature of $25^{\circ} \mathrm{C}$ followed by 5 and $35^{\circ} \mathrm{C}$ respectively.

\section{DISCUSSION}

P. guilliermondii strain $\mathrm{Z} 1$ is a new potential biocontrol agent recently isolated from orange fruit surface and selected for its great effectiveness against $P$. italicum and $P$. digitatum (El Guilli et al., 2009; Lahlali et al., 2011). The ability of this strain to suppress both pathogens was evaluated at semi-commercial scale and showed a reduction of $P$. italicum incidence by more than $90 \%$ (El Guilli et al., 2009) and seems to act through its ability to colonize the apple surface, which explains the usefulness of tracking the population dynamics of the strain Z1 after its application on citrus fruit. The classical method for quantification of a particular BCA consists of counting 


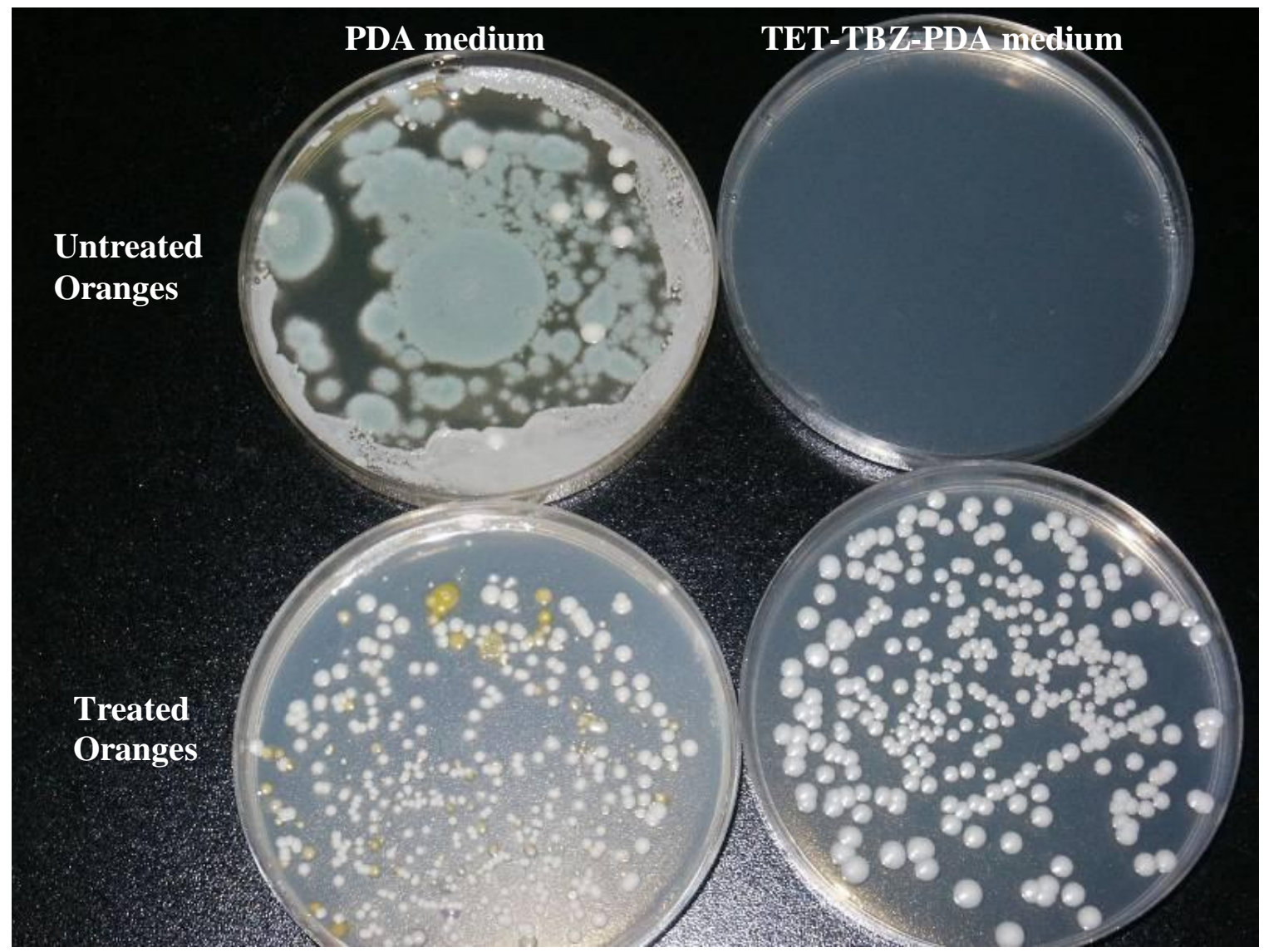

Figure 1. Efficiency of semi-selective medium TET-TBZ-PDA in comparison with standard PDA medium: Plating of wash fruit water treated or not with $P$. guilliermondii strain $Z 1$.

colony-forming units (CFU) on a selective or semiselective solid medium (Leibniger et al., 1997; Lima et al., 1999; Johnson et al., 2000; Mercier and Wilson, 1995; Usall et al., 2001). This method has the advantage that only living microorganisms are detected. To monitor the population progress of this strain when applied pre or postharvest on orange fruit surface, a semi-selective medium is required and recommended in preventing the development of indigenous microorganisms and allow only the detection and enumeration of strain Z1. Therefore, different fungicide and antibiotic combinations that could be combined with the basal potato dextrose medium have been assayed. De Clercq et al. (2003) have developed a semi-selective medium for both antagonistic yeasts, Pichia anomala strain $\mathrm{K}$ and Candida oleophila strain $\mathrm{O}$, consisting of three fungicides (thiram, carbendazim and diethofencarb) and an antibiotic hygromycin B. Results from the preliminary first screening shows that thiabendazole (TBZ) is a more appropriate fungicide because it was less toxic than others such as imazalil, thiophanate methyl or thiram with higher efficiency when applied at $60 \mathrm{mg} / \mathrm{L}$. Similarly, the antibiotic tetracyclin (TET) proved to be more efficient than hygromycin, ampicillin and chloramphenicol. Ten combinations give higher selectivity against $P$. italicum, $P$. digitatum, laboratory air microflora and washing water pathogens from orange surface that were retained. Among them, one combination of TBZ $(60 \mathrm{mg} / \mathrm{L})$ and tetracyclin (1000 $\mathrm{mg} / \mathrm{L})$ which allowed higher efficiency (99\%) and selectivity (Figure 1) was selected as semi-selective medium of this strain. This selective medium gave the same population density as compared to PDA alone. To our knowledge, the semi-selective medium (TET05+TBZ04) using PDA as the basal medium is the first semi-selective medium developed for the isolation and quantification of $P$. guilliermondii strain Z1. This medium completely inhibits the natural microorganisms present on orange surface and postharvest citrus fruit pathogens and did not permit the development of conta-minants from ambient air. Jijakli et al. (1999) underlined that the development of a monitoring method for tracking the population dynamic of BCA on the fruit surface is of particular interest because the efficiency of the yeast is closely related to its colonization. Such findings have been confirmed by Lahlali et al. (2008) who reported that a yeast density of 1 $\times 10^{4} \mathrm{CFU} / \mathrm{cm}^{2}$ of apple fruit surface is required to reach 


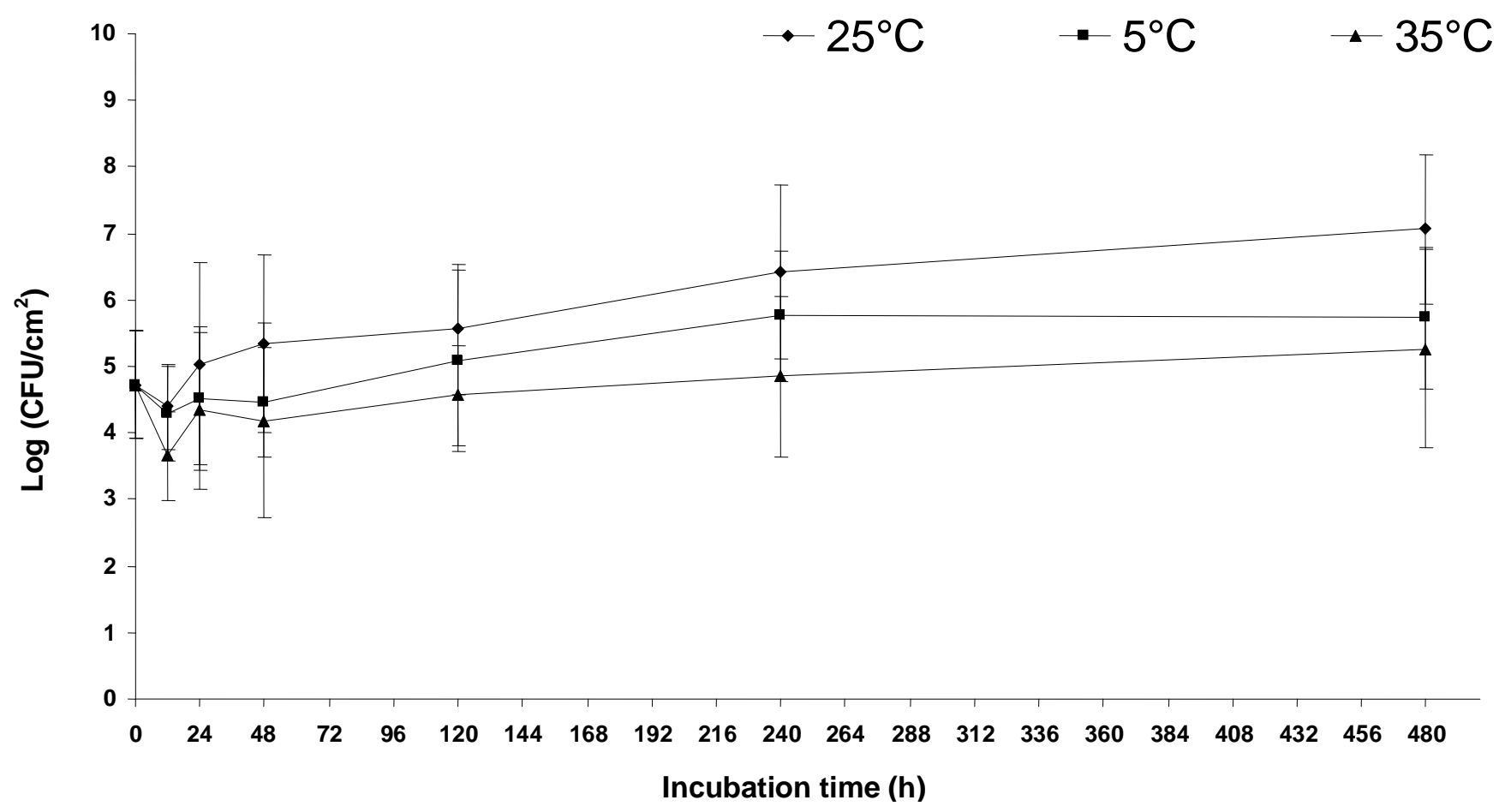

Figure 2. Impact of incubation temperature $\left(5,25\right.$ and $\left.35^{\circ} \mathrm{C}\right)$ on population size of $P$. guilliermondii strain $\mathrm{Z1}$ on orange fruit surface.

a higher protection against $P$. expansum during storage. Therefore, a semi-selective medium allowed growers to determine which concentration of BCA could be used to obtain complete reduction of the rot incidence on fruit rot. Lahlali et al. (2008) demonstrated that the initial concentration of application is a crucial factor limiting the effectiveness of antagonistic yeast when applied at preharvest and the density of yeast on apple surface was significantly dependant on environmental factors and with its initial concentration of application.

Different wash buffers have been used in literature to recover the population of antagonistic yeast on fruit surface (Beuchat and Pitt, 1990; Chand-Goyal et al., 1998; Leibniger et al., 1997; Lima et al., 1999; Johnson et al., 2000; Usall et al., 2001). In this work, we have used KBTP as a wash buffer. This wash buffer has been largely cited for higher recovery efficiency with more than $99 \%$ of yeast cells from the treated apple surface (De Clerq et al., 2004; Lahlali et al., 2008; Pujol et al., 2004). Having selected the semi-selective medium for the strain $Z 1$, we conducted a study on the impact of temperature on growth of strain $\mathrm{Z1}$ in in vivo conditions. Three temperatures $\left(35,25\right.$ and $\left.5^{\circ} \mathrm{C}\right)$ were tested. The curve representing the population density of this strain at each temperature according to the incubation times followed the same shape and suggested that this strain needs adaptation time to overcome the heat stress before entering into the exponential growth phase which may explain the drop of populations of strain $\mathrm{Z} 1$ after $24 \mathrm{~h}$ of incubation. Teixido et al. (1999) evaluated the population dynamic of Candida sake at 1 and $25^{\circ} \mathrm{C}$ on apple surface and reported that the population density of this antagonistic yeast dropped during the first $24 \mathrm{~h}$ after its application, but it was gradually increased to reach a maximum after one month of incubation. Similarly, De Clercq et al. (2003) underlined that the population of antagonistic yeast $P$. anomala dropped by $30 \%$ after one week of incubation on apples at 25 and $4^{\circ} \mathrm{C}$. Our strain $\mathrm{Z} 1$ shows an increase in density over time range required for long-term protection of citrus fruits during storage.

In conclusion this medium could not distinguish our biocontrol agent from other closely related species. However, this classical method for quantification of BCA based on counting colony forming units on Petri dish on a semi-selective medium have the advantage that only living microorganisms are quantified. Additionally, this method is cheaper than other molecular techniques based on SCAR markers and could be an efficient routine laboratory method used for evaluation of population size of $P$. guilliermondii strain $\mathrm{Z} 1$ or other closely related species at different environmental conditions, especially in developing countries where molecular techniques are rarely available and too expensive.

\section{ACKNOWLEDGEMENTS}

The authors are grateful to Direction Générale de la Coopération au Développement-Commission Universitaire pour le Développent (DGCD-CUD) for the financial support in the case of Projet interuniversitaire ciblé (PIC) Morocco project. 


\section{REFERENCES}

Beuchat LR, Pitt JI (1990). Influence of solute, pH, and Incubation Temperature on Recovery of Heat-Stressed Wallemia sebi Conidia. Appl. Environ. Microbiol. 56:2545-2550.

Chand-Goyal T, Eckert JW, Droby S, Atkinson K (1998). A method for studying the population dynamics of candida oleophila on oranges in the grove, using a selective isolation medium and PCR technique. Microbiol. Res. 153:256-270.

Citrus Commodity Notes (2005). Citrus Commodity Notes 2005. Developments in international citrus trade in 2004-2005.

De Clercq D, Cognet S, Pujol M, Lepoivre P, Jijakli MH (2003). Development of a SCAR marker and a semi-selective medium for specific quantification of Pichia anomala strain $\mathrm{K}$ on apple surface. Postharvest Biol. Technol. 29:237-47.

El Guilli M, Achbani E, Fahad K, Jijakli MH (2009). Biopesticides: alternatives à la lutte chimique in Symposium international 'Agriculture durable en région Méditerranéenne (AGDUMED', rabat, Maroc (14-16 mai), 266-280.

El Guilli M, Ibriz M, Lahlali R, Jijakli MH (2012). Effects of Temperature and Relative Humidity on the In Vitro and In Vivo Radial Growth of Penicillium italicum and on the Biocontrol Activity of Pichia guilliermondii, Strain Z1. Acta Hortic. 905:233-240.

Jijakli MH, Lepoivre P, Grevesse C (1999). Yeast species for biocontrol of apple postharvest disease : an encouraging case of study for practical use. In: Mukerji KG, Chamola BP, and Upadhyay RK, eds. Biotechnological approaches in biocontrol of plant pathogens. Klumer Academic / Plenum Publishers, New york, p. 31-49.

Johnson KB, Stockwell VO, Sawyer TL, Sugar D (2000). Assessment of environmental factors influencing growth and spread of Pantoea agglomerans on and among blossoms of pear and apple. Phytopathology 90:1285-94.

Lahlali R, Hamadi Y, El Guilli M, Jijakli MH (2011). Efficacy assessment of Pichia guilliermondii strain Z1, a new biocontrol agent, against citrus blue mould in morocco under the influence of temperature and relative humidity. Biol. Control 56:217-224.

Lahlali R, Massart S, Serrhini MN, Jijakli MH (2008). A Box-Behnken design for predicting the combined effects of relative humidity and temperature on antagonistic yeast population density at the surface of apples. Int. J. Food Microbiol. 122: 100-108.

Lahlali R, Serrhini MN, Jijakli MH (2004). Efficacy assessment of Candida oleophila (strain O) and Pichia anomala (strain K) against major postharvest diseases of citrus fruits in Morocco. Commun. Agric. Appl. Biol. Sci. 69:601-609.

Lahlali R, Serrhini MN, Jijakli MH (2005). Development of a biological control method against postharvest diseases of citrus fruits. Commun. Agric. Appl. Biol. Sci. 70:47-58
Leibniger W, Breuker B, Hahn M, Mendgen K (1997). Control of postharvest pathogens and colonization of the apple surface by antagonistic microorganisms in the field. Phytopathology 87:1103-10.

Lima G, Arru S, De Curtis F, Arras G (1999). Influence of antagonist, host fruit and pathogen on the biological control of postharvest fungal diseases by yeasts. J. Ind. Microbial. Biotech. 23:223-9.

Mercier J, Wilson CL (1995). Effect of wound moisture on the biocontrol by Candida oleophila of gray mold rot (Botrytis cinerea) of apple. Postharvest Biol. Technol. 6:9-15.

Palou L, Usall J, Munoz A, Smilanick JL, Vinas I (2002). Hot water, sodium carbonate, and sodium bicarbonate for the control of postharvest green and blue molds of Clementine mandarins. Postharvest Biol. Technol. 24:93-96.

Pujol M, De Clercq D, Cognet S, Lepoivre P, Jijakli MH (2004). Monitoring system for the biocontrol agent Pichia anomala strain $\mathrm{K}$ using quantitative competitive PCR-ELOSA. Plant Pathol. 53 :103109

Taqarort N, Echairi A, Chaussod R, Nouaim R., Boubaker H, Benaoumar AA, Boudyach EH (2008). Screening and identification of epiphytic yeasts with potential for biological control of green mold of citrus fruits. World. J. Microbiol. Biotechnol. 24:3031-3038.

Teixido N, Usall J, Vinas I (1999). Efficacy of preharvest and postharvest Candida sake biocontrol treatments to prevent blue mould on apples during cold storage. Int. J. Food Microbiol. 50:203210

Usall J, Teixido N, Torres R, Ochoa de Eribe X, Vinas I (2001). Pilot tests of Candida sake (CPA-1) applications to control postharvest blue mold on apple fruit. Postharvest Biol. Technol. 21:147-56.

Zhang H, Zheng X, Xi Y (2005). Biological control of postharvest blue mold of oranges by Cryptococcus laurentii (Kufferath) Skinner. BioControl 50:331-342. 